\title{
Matching and oddity conditional discrimination in the goldfish as avoidance responses: Evidence for conceptual avoidance learning
}

\author{
D. J. ZERBOLIO and JOEL L. ROYALTY \\ University of Missouri, St. Louis, Missouri
}

\begin{abstract}
Goldfish, trained in the avoidance shuttlebox with a variant of the linear discrimination procedure, learned to conditionally discriminate between color signals, both for the matching $(\mathrm{M})$ and oddity $(\mathrm{O})$ criterion forms. Transfer to assess the possibility of concept learning was also tested. In original learning, oddity-trained groups learned faster and reached higher conditional discrimination performance levels than did matching-trained groups. In transfer, various groups were tested with the same criterion (MM or OO) or a shifted criterion (MO or $O M)$, and half of each group retained the same color signals and the remaining half had its color signals changed in transfer. Groups with the same criterion in original learning and transfer (MM or OO), regardless of signal colors, showed comparable positive transfer. Groups with their criterion shifted between original learning and transfer (MO or OM) showed comparable negative transfer, regardless of signal colors. Since both positive- and negative-transfer effects were independent of signal colors, it is clear that what was learned for one set of signal colors transferred to at least one other signal-color set. These findings are consistent with the interpretation that goldfish learned the original conditional discrimination at a conceptual level, and learned about the general matching or oddity relationships between colors, rather than about a specific set of colors.
\end{abstract}

Discrimination learning is typically accomplished by exposing an animal to two different signal or stimulus conditions and systematically and differentially reinforcing responses to each signal. With training, the animal usually learns to prefer one signal condition over the other, presumably because it prefers one signal-specific response consequence over the other. One cannot help being impressed with the discriminative learning capacity of a wide variety of animals (e.g., Mackintosh, 1974). However, if one carefully examines the cited studies, it is clear that the vast majority are predicated on food/ food-omission reinforcement. Very few, if any, are the result of shock/shock-omission reinforcement. There seems to be no good reason for this discrepancy except that animals do not seem to learn to discriminatively choose between signals differentially paired with shock and shock-omission reinforcers. As a result, very little is known about shock/shock-omission reinforced discrimination learning. However, there are exceptions to this.

Recently, goldfish have been shown to be capable of learning to choose between color signals that are differentially and systematically associated with shock and shock omission. This has been demonstrated in the Y-maze with the use of a simultaneous presentation procedure (Zerbolio \& Wickstra, 1979, 1980) and in the shuttlebox with the use of the linear presentation pro-

The author's mailing address is: Department of Psychology, University of Missouri, St. Louis, Missouri 63121. cedure (Zerbolio, 1981; Zerbolio \& Royalty, 1982). There are some differences in the avoidance-response requirements for these two different apparatuses and procedures. In the $Y$-maze, the required response is the choosing of a specific area or alley in which the shockomission-paired signal is displayed. Position in the maze becomes crucial as the goldfish changes the color state of its immediate surrounds by selecting the appropriate alley and swimming to it. In the shuttlebox, with the linear presentation procedure, shuttling responses change the color or illumination state of the entire shuttlebox. What is crucial is not the animal's position in the shuttlebox, but rather the color state of the entire apparatus at shock-delivery times. If the shock-omission signal prevails at shock time, shock is omitted. If any other color or illumination state prevails, shock is delivered as programmed. To avoid shock, goldfish learn to control the color state of the entire apparatus by responding, rather than by swimming to a specific part of it. What is surprising about the results with either of these procedures is the ease and efficiency with which goldfish learn a discrimination response to avoid shock. This performance raises a question about the discrimination learning capacity of goldfish in procedures employing shock/ shock-omission reinforcement procedures.

This question can be approached in at least two ways. One is to increase the complexity of stimulus signals within the signal-specific discrimination procedure to see what kinds of different signals the goldfish can discriminate (e.g., Bowman \& Sutherland, 
1969). The other is to increase the complexity of the task by asking the goldfish to learn the relationship between signals. This latter approach is best exemplified by the conditional discrimination approach.

In the conditional discrimination approach, the initial requirement is to teach the animal to choose a signal that is like (matches) or is different from (oddity) a current-trial standard signal. Once the original task is mastered, the animal is tested in a transfer task in which all signals are changed. In transfer, if the animal continues to select the matching (or odd) signal from the "new" available choices, it is assumed the animal has learned the concepts of matching or oddity.

An example of this approach with color signals is Zentall and Hogan's (1974) work. However, Carter and Eckerman (1975) challenged the "concept learning" conclusion by pointing out that Zentall and Hogan's (1974) shifted and nonshifted birds did not differ on the 1st day of transfer. In answer to this criticism, Zentall and Hogan (1978) trained pigeons on an initial brightness conditional discrimination, using "negative instances," and then on a color conditional discrimination. In transfer to a second color conditional discrimination, they found substantial transfer on the 1st day of training, a result that provided additional support for their earlier concept-learning conclusion.

Goldfish have been shown to be capable of learning a conditional discrimination both with food/food-omission reinforcement procedures (Goldman \& Shapiro, 1979) and, in a Y-maze, with shock/shock-omission reinforcement procedures (Wickstra, 1980). However, Goldman and Shapiro (1979) did not attempt a transfer test, and Wickstra (1980) was unable to develop an appropriate transfer test procedure to assess matching and oddity performance as concepts.

It is possible to use the shuttlebox to administer a conditional discrimination problem to goldfish. If provisions for different trial standard signals in addition to different response-contingent signals are made, all of the necessary signal requirements would be available. The animal's task would simply be to select the signal from the available choices that "matches" or is "odd from" the current trial standard. The present study aimed to determine if goldfish could learn a conditional discrimination in the shuttlebox, and, if they could, to test them in a transfer procedure with entirely new color signals to determine if they had acquired the matching or oddity discriminations as concepts.

\section{METHOD}

\section{Subjects}

Forty-eight 5-6-cm-long goldfish, obtained from Ozark Fisheries, served as subjects. They were housed in $7.5 \times 11.4$ $\times 12.5 \mathrm{~cm}$ deep individual holding tanks. The water in the holding tanks was well filtered and aerated. About $25 \%$ of the water was removed daily by siphoning to remove debris, and then replaced. The tanks were scrubbed weekly, and all the water was replaced. Temperature $\left(21^{\circ} \pm 1^{\circ} \mathrm{C}\right)$ and $\mathrm{pH}(7 \pm .1)$ were held constant. The goldfish were fed daily.

\section{Apparatus}

Four $29.2 \times 11.4 \times 11.4 \mathrm{~cm}$ deep aquatic shuttleboxes, similar to those described by Horner, Longo, and Bitterman (1961), were used. In the center of each shuttlebox was a 6.35-cm-high hurdle, with a 9-cm flat top, and 45-deg sloping ramps into deep wells on each end. The water was maintained at $2 \mathrm{~cm}$ deep over the flat top of the hurdle at all times. The water in the shuttleboxes was constantly aerated, and about $25 \%$ was siphoned off daily to remove debris and then replaced. The shuttleboxes were emptied weekly, cleaned, and refilled. Shuttle activity was monitored by photocells at the ends of the flat top portion of the hurdle. Photocell light sources were $2.5-\mathrm{V} \mathrm{dc}$ prefocused penlight bulbs, run at $1.5-\mathrm{V}$ ac to extend bulb life. A clear plastic lid was placed on top of each shuttlebox during testing. Different color signals were provided by $7-\mathrm{W} 110-\mathrm{V}$ ac red, green, blue, and yellow Christmas tree bulbs. Three light sources were used to indicate intertrial interval (ITI), trial standard color, and response-contingent color illumination. A fixture placed on top of the clear lid cast light directly down into the box and provided the white ITI. Except for a $5-\mathrm{cm}$ strip from side to side that allowed hurdle illumination from underneath, the bottom of each shuttlebox was painted flat black. A fixture placed directly under the shuttlebox shone directly upward, illuminating the hurdle, and provided the trial standard color. In addition, the hurdle was sandblasted to help diffuse trial standard illumination. A black plastic baffle that extended from under the hurdle to the bottom of the tank separated the ends of the shuttlebox visually, so that a fish in the well at one end could see the hurdle and the occupied end, but could not see the other end unless it crossed the hurdle. Fixtures placed at the ends of each shuttlebox provided response-contingent color illumination. The bottom and end lamp fixtures held differently colored, individually programmable bulbs, allowing for changes in the trial standard or response-selectable colors.

Translucent .16-cm white plastic plates were placed between each light source and the shuttlebox to diffuse illumination evenly. The measured spectral peaks of the red, green, blue, and yellow Christmas tree bulbs were $620 \pm 4,517 \pm 2,474 \pm 2$, and $586 \pm 2 \mu$, respectively. Obvious brightness differences between differently colored bulbs were initially adjusted by placing a diode in the $110-\mathrm{V}$ ac power line for the red and yellow color sources, and using two green and two blue bulbs to generate the green and blue signals. Following these adjustments, the luminances for each source were measured (through the water in the shuttletank) with a Macbeth illuminometer positioned $10 \mathrm{~cm}$ above the center hurdle. The luminances of hurdle trial standard colors were measured through $2 \mathrm{~cm}$ of water, and those for the end lights (a reflecting mirror was used), through a total path of $15 \mathrm{~cm}$ of water. For the center hurdle, measured luminances for red, blue, green, and yellow sources were 8 to 13,5 to 10 , 6 to 15 , and 4 to $10 \mathrm{~mL}$, respectively. For the end-light color sources, measured luminances for the red, blue, green, and yellow sources were 1 to 4,1 to 2,1 to 4 , and 1 to $4 \mathrm{~mL}$, respectively. In general, as much variability in luminance between sources of the same color as between sources of different colors was observed. The brightness of the white ITI overhead light was also attenuated with a series diode. Shock, in 200-msec pulses at $7 \mathrm{~V}$ ac $(.69 \mathrm{~V} / \mathrm{cm})$, was delivered by $28 \times 10.2 \mathrm{~cm}$ 22-ga stainless steel plates fixed to the inside vertical side walls of the shuttlebox.

\section{Procedure}

The experiment was divided into two phases: an originallearning phase and a transfer phase. Each phase lasted 30 training days, for a total of 60 days in all. The goldfish were run 40 trials/day, 5 days/week, and were rested on weekends. Trials were separated by a 60-sec variable-interval ITIs, ranging from 30 to $90 \mathrm{sec}$. Different illumination or colors indicated the ITI, trial start, current trial standard, and response-contingent color state to the animal. White overhead illumination indicated the ITI. Termination of the white ITI light, rendering the shuttle- 
box dark, indicated the start of a trial. Two seconds later, the current-trial standard color was indicated by colored light from under the center hurdle. The specific color of the trial standard was determined randomly (Gellerman series), so that for each day's 40 trials, 20 were of one color and the remaining 20 were of the other color. A response was defined as a goldfish's swimming from the end occupied at trial onset, crossing the hurdle, and breaking the farthest photobeam. The goldfish's first response on a trial turned on, at random, one or the other of the response-contingent colors in the fixtures at the ends of the shuttlebox. Both ends were illuminated with the same color (e.g., both red or both green). Additional responses caused the color emanating from the end fixtures to alternate (red to green or vice versa), and alternation occurred with every response of the trial after the initial response. During the trial period, the color of the trial standard (hurdle) remained constant, but the color state of the end lamps (e.g., dark, red, or green) was controlled by the goldfish via response-contingent programming.

Shock, in 200-msec pulses, was scheduled to occur at 12,14 , and $15 \mathrm{sec}$ after trial onset. If, however, the relationship between the color of the trial standard color and the end-lamp response-contingent color met the procedural criterion, shock was omitted. For the matching procedure, the criterion was a match (same color) of end and hurdle lamps. For the oddity procedure, the criterion was a difference (in color) between the end and hurdle lamps. The instrumentation compared the color relationship between hurdle and end lamps at each of the three scheduled shock times and administered shock accordingly. The goldfish had to produce and maintain the proper color relationship between hurdle and end lamps for all three shock times to be credited with an avoidance response. The three-shock procedure ailowed the goldfish to prevent (escape) further shock on a trial if it had failed to meet the proper criterion at $12 \mathrm{sec}$. It also provided a mechanism for detecting an animal responding randomly. Randomly responding fish might miss the first shock pulse by chance, but were much less likely to miss all three pulses because each response alternated the end-lamp colors. If, by chance, the animal met the procedural criterion at $12 \mathrm{sec}$ and continued to respond, the relationship between hurdle and end lamps changed, producing shock delivery at one of the other shock times. The staggered intershock interval $(12$ to $14 \mathrm{sec}$, 14 to $15 \mathrm{sec}$ ) was also designed to facilitate detecting random response and not crediting it as avoidance. The goldfish could miss the first shock, respond, change the color relationship, and correct by responding again to reestablish the proper color relationship, providing the correction was completed before the next shock. Also, if the goldfish receiving a shock pulse responded within the 200-msec duration and produced the proper color relationship between hurdle and end lamps, the shock was terminated. Such correction or shock-termination responses were very rare. All color illumination occurring during the trial was extinguished at $15.2 \mathrm{sec}$, and the white overhead ITI illumination was reestablished. At the end of each trial, two items were recorded: whether or not the goldfish responded before the first shock at 12 sec (trials with response, or TwR measure) and, given TwR, whether the goldfish avoided (met the procedural criterion at all three shock times). At the end of each day's testing, the number of TwR and the number of avoidances for each fish were recorded and used to calculate a further performance measure, the discrimination index (DI).

The DI measure, used earlier by Zerbolio and Royalty (1982), was $[(S+)-(S-)] / 40$, where $S+$ was the number of trials in which the animal avoided (chose the shock-omissionpaired signal), $\mathrm{S}-$ was the number of trials in which the animal received shock (chose the shock-paired signal), and where $(S+)+$ $(S-)=T w R$. In the present study, $S+$ was the number of $T w R$ in which the animal met the specific procedural criterion requirement (and thus avoided), and $S-$ was the number of TwR in which shock occurred. As before, $\left(\mathrm{S}_{+}\right)+\left(\mathrm{S}_{-}\right)=$TwR. The DI measure has limits of \pm 1.00 , and is a perfect 1.00 only if the animal responds and discriminates (avoids) on all 40 trials. If the animal responds before shock on all trials, but incorrectly discriminates on each trial, the DI measure will be -1.00 . DI values of zero indicate no discrimination. Furthermore, since the denominator is 40 (the number of daily trials), the index differentiates between animals responding on just a few trials and those responding on many or all trials.

Original learning (OL). In OL, 48 goldfish were split randomly into two groups of 24 each. One group was trained with the matching (M) criterion and the other group with the oddity (O) criterion. Of each criterion group, half $(n=12)$ received red/green signal colors and the remainder $(n=12)$ received blue/yellow signal colors. Thus, signal colors (red/green vs. blue/ yellow) and criteria [matching (M) vs. oddity (O)] were completely balanced in original learning. All goldfish were trained for 30 days.

Transfer testing. Transfer testing called for shifting the original-learning criterion for half the fish but not for the remainder. Signal colors were changed (C) for half and remained the same $(S)$ for the remainder. Not all fish were tested in transfer. Four matching- and 1 oddity-trained goldfish did not learn the original problem and were excluded from further study. Of the remainder, the 16 best matching- and 16 best oddity-performing goldfish were identified with the DI measure and were used in transfer. The 16 matching goldfish were split into four groups of $n=4$ each, with each group balanced for original learning colors, and were equated for original-learning performance. The same procedure was followed for the 16 oddity-trained fish. In transfer, half retained their originallearning criterion (matching-matching or oddity-oddity, $\mathrm{MM}$ or $O O)$ and half had their original-learning criterion shifted (matching-oddity or oddity-matching, MO or OM). Half retained the same $(S)$ signal colors experienced in original learning and half had their signal colors changed $(C)$ in transfer. This generated a factorial arrangement of original-learning criterion (M vs. O) $\times$ transfer criterion (M vs. O) $\times$ signal colors in transfer (S vs. C). The eight different groups generated by this arrangement were MMS, MMC, MOS, MOC, OOS, OOC, OMS, and OMC. Color changes were easily accomplished. Recall that four shuttleboxes were used, two with red/green and two with blue/yellow signals. The $\mathrm{C}$ goldfish were tested in transfer in one of the "other color" shuttleboxes. To equate the possible effects of shuttlebox change, the $S$ groups were also tested in a different shuttlebox, but one with the same colors as they had had in original learning. The entire procedure, including the exclusion of poorly performing animals from transfer testing, is comparable to that used by Zentall and Hogan (1974), but with the addition, in the present study, of balancing for the colors used in original learning. All other transfer-testing conditions, procedures, and measures were identical to those used in original learning.

\section{RESULTS AND DISCUSSION}

The data for 30 days of original learning and 30 days of transfer testing were separated to facilitate analysis, presentation, and discussion. All figures present data over 10 3-day blocks. The data were analyzed both over 30 days of training and for 103 -day blocks, but no essential differences were found. Thus, only the 10 3-dayblock analyses are presented.

\section{Original Learning}

The results of original learning, for both matchingand oddity-contingency criteria and for the 48 original and 32 selected goldfish tested in the transfer procedure, are shown over 10 3-day blocks in Figures 1 and 2. Figure 1 shows the mean percent TwR and avoidances 
(avoid) and also shows the chance avoidance measure, $\mathrm{E}$ (avoid), for each group. The chance avoidance measure, $E$ (avoid), is half of the trials with response because a randomly responding goldfish would be expected to generate a current trial standard/response contingent color relationship condition meeting the matching or oddity criterion on half of its TwR, by chance. Thus $\mathrm{E}$ (avoid) is $.5 \times$ TwR and is included in Figure 1 for comparison purposes only. Figure 2 shows the mean DI performance for 10 3-day blocks for the same groups. In addition, Figure 2 has .01 significance intervals about zero discrimination for both the 48 original and 32 selected goldfish tested in transfer.

Figures 1 and 2 show that, with training, goldfish learn to respond on an increasing number of trials (TwR), avoid more often, and respond discriminitively (DI). Furthermore, the oddity-criterion goldfish perform better than do the matching-criterion goldfish on all measures. These impressions were confirmed by the statistical analysis for the initial 48 goldfish, which found significant increases in TwR, avoidances, and DIs over training blocks $[\mathrm{Fs}(9,414)=291.65,169.69$, and 76.17 , respectively, all ps $<.011$. The 24 oddity-trained goldfish were significantly superior to the 24 matchingtrained goldfish on each of the TwR, avoidances, and DI measures as well $[\mathrm{Fs}(1,46)=8.45, \mathrm{p}<.02,9.39, \mathrm{p}<.01$, and $8.99, \mathrm{p}<.01$, respectively]. In addition, interactions between training blocks and matching-/odditycriterion procedures were found for the original 48 goldfish on both the avoidance and DI measures $[\mathrm{Fs}(9,414)$ $=2.08$ and 2.01 , respectively, both ps $<.05$ ] . To understand these interactions, matching and oddity groups were compared for each 3-day block. For the avoidance measure, matching and oddity groups did not differ on the first two 3-day blocks, but oddity-trained goldfish avoided on significantly more trials from the third

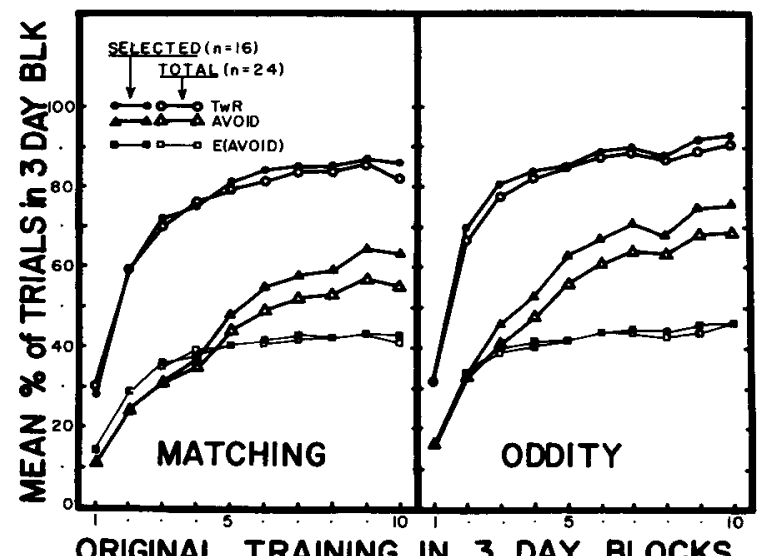

Figure 1. The mean percent trials with response (TwR), avoidances, and E(avoid), in 3-day blocks, for the original 24 matching- and 24 oddity-criterion-trained goldfish and for the selected 16 matching- and 16 selected oddity-trained goldfish used later in transfer. The $E$ (avoid) measure is TwR $\times .5$ and represents chance avoidance.



Figure 2. The mean discrimination index (DI), in 3-day blocks, for the original 24 matching- and 24 oddity-trained goldfish and the 16 matching- and 16 oddity-trained animals selected for transfer testing. A .01 interval, from zero or no discrimination, is incorporated into the figure to show above-chance discrimination for groups with 24 and 16 fish.

3-day block on $[\operatorname{ts}(460)=2.23$ or greater, all ps $<.05]$. For the DI measure, matching and oddity groups did not differ until the fourth 3-day block, but oddity groups discriminated significantly better on the DI measure from then on $[\operatorname{ts}(460)=2.58$ or greater, all ps $<.02]$.

Comparable results were found for the 32 goldfish selected for transfer training. For these selected goldfish, significant increases in TwR, avoidances, and DIs were observed with training $[\mathrm{Fs}(9,216)=275.59$, 190.74 , and 94.78 , respectively, all ps $<.01$ ] . On each of the TwR, avoidances, and DI measures, the odditytrained goldfish were superior to the matching-trained animals $[\mathrm{Fs}(1,24)=5.16, \mathrm{p}<.05,8.48, \mathrm{p}<.01$, and $9.06, \mathrm{p}<.01$, respectively]. For the 32 selected goldfish, no interactions were observed.

One additional factor was included in the 32 selectedfish analyses. The selected matching- and oddity-trained fish were split into four subgroups of $n=4$ prior to transfer testing. These splits were included as a factor in the selected-fish analyses. However, no effects (neither main nor interactions) were found involving splits, which indicated that the four groups, within the matching and oddity criteria, were alike prior to transfer testing.

From these data, it is clear that goldfish, trained with either the matching- or oddity-criterion form of the conditional discrimination procedure in the shuttlebox, learned to respond on an increasing number of trials and avoided more often, but none of these analyses directly addresses the question of whether their observed avoidance rates exceed chance. To answer this question, a .01 confidence interval from chance or zero DI was calculated for both the 48 original and the 32 selected goldfish and is incorporated into Figure 2. From this figure, it is clear that both the matching- and odditytrained groups exceed chance avoidance discrimination. 
The oddity-criterion groups exceeded chance DI levels by the fourth 3-day block and the matching-criterion groups by the sixth 3-day block. Clearly, goldfish learn to select matching or odd color signals in the shuttlebox to avoid shock.

\section{Transfer Testing}

If goldfish conceptually learn the conditional discrimination to avoid shock in original learning, their transfer-testing performances should be dependent on the criterion contingency in the transfer-testing situation and independent of the signal colors. If the criterion contingency is the same in transfer testing as in original learning, positive transfer, or avoidance discrimination well above chance, would be expected. If, however, the criterion contingency is shifted between original learning and transfer testing, then negative transfer, or avoidance discrimination well below chance, would be expected. In either case, the transfer effects are expected to be independent of whether the signal colors in transfer are the same (S) or changed (C) from those used in original learning. To facilitate testing for conceptual learning, transfer groups were arranged in pairs, such that one member of each pair retained the same (S) signal colors it had had in original leaming and the other member of each pair had its signal colors changed (C) in transfer testing. Both members of each pair shared the same original-learning/transfer-testing criterion-contingency histories, that is, match-match (MM), oddity-oddity (OO), match-oddity (MO), or oddity-match (OM). The transfer-testing performances of pairs sharing the same original-learning/transfer-testing criterion-contingency histories appear in the various panels of Figures 3 and 4. Figure 3 shows the mean percent TwR, avoidance, and E(avoid) performances, and Figure 4 shows the mean DI index, including a .01 chance discrimination interval, for the 10 3-day blocks of transfer testing.

Figures 3 and 4 clearly show that groups retaining the same criterion contingency in transfer testing (MM and OO) maintained $T w R$, avoidance, and DI performance during transfer testing, regardless of whether their signal colors were the same (MMS and OOS) or changed (MMC and OOC) in transfer. The effects of shifting the criterion contingency are just as clear. When the criterion contingency was shifted between original learning and transfer testing (MO or OM), TwR rates remained relatively high, whereas avoidance and DI performances dropped well below chance discrimination levels, indicating substantial negative transfer. Furthermore, negative transfer occurred regardless of whether the signal colors were the same (MOS and OMS) or changed (MOC and OMC) in transfer testing. In addition, both the positivetransfer effects (MMS, MMC, OOS, OOC) and negativetransfer effects (MOS, MOC, OMS, OMC) were significantly different from chance expectancies for all groups. This is clearly shown in Figure 4 , in which the first 3-day-block transfer-test performances on the DI measure for all groups are outside the .01 chance discrimina-

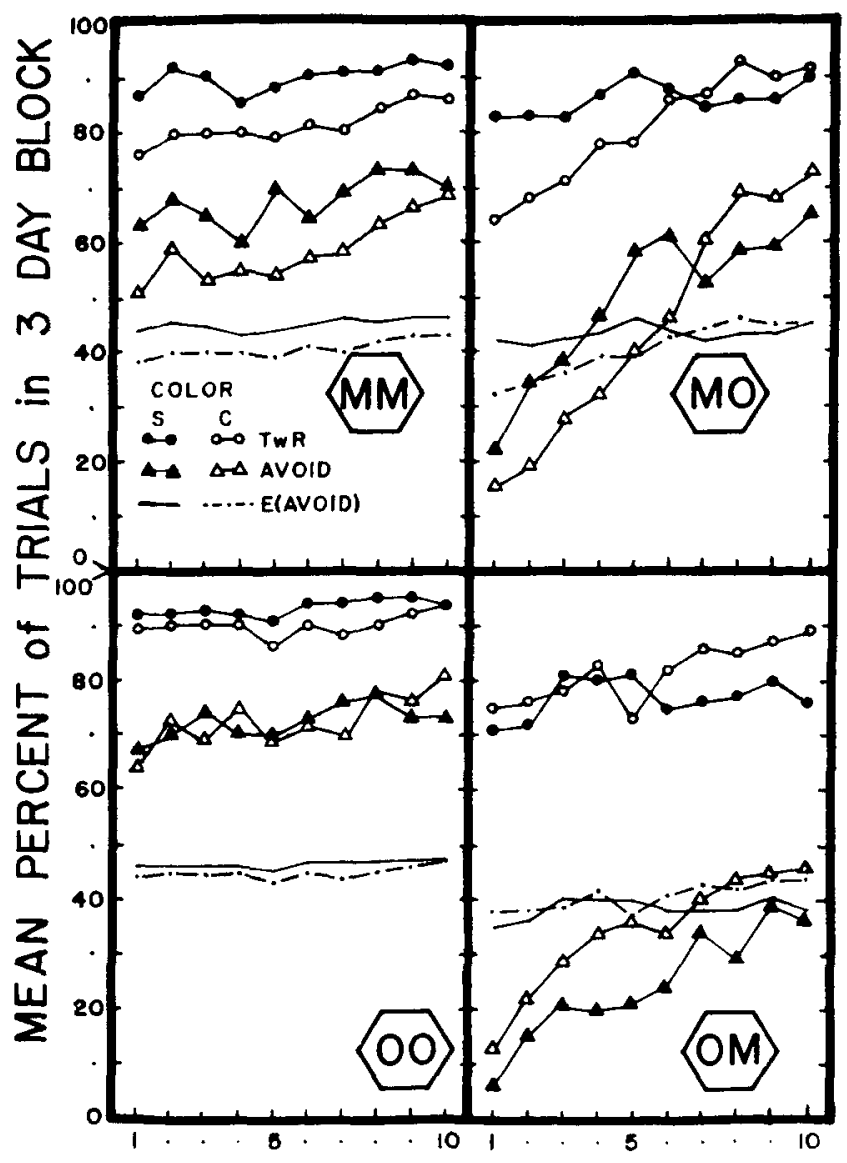

TRANSFER TRAINING in 3 DAY BLKS

Figure 3. The mean percent trials with response (TwR), avoidances, and E(avoid), in 3-day blocks, for all groups in transfer testing. Panels show groups having had identical originallearning/transfer-testing criterion-contingency histories, but differing in having their signal colors the same (S) or changed (C) in transfer testing from those experienced in original learning.

tion confidence interval and are in the predicted direction. It is also clear that the difference between the matching and oddity forms of the conditional discrimination problem observed in original learning also occur in transfer. The OOS and OOC transfer groups appear to perform better in transfer than do the MMS and MMC groups, and the MOS and MOC groups appear to learn the shifted transfer task to above-chance discrimination levels by the end of transfer testing, whereas the OMS and OMC groups do not. All but one of these impressions were confirmed by the statistical analyses.

The initial statistical analyses, for each of the TwR, avoidances, and DI measures, were four-way repeatedmeasures analyses of variance (ANOVAs), with criterion in original learning ( $M$ vs. $O)$, criterion in transfer testing ( $M$ vs. 0 ), and signal-color condition in transfer [the same or changed in transfer ( $\mathrm{S}$ vs. C)] as betweengroups factors and 3-day blocks of training as the repeated-measures factor. In each of the large analyses, 


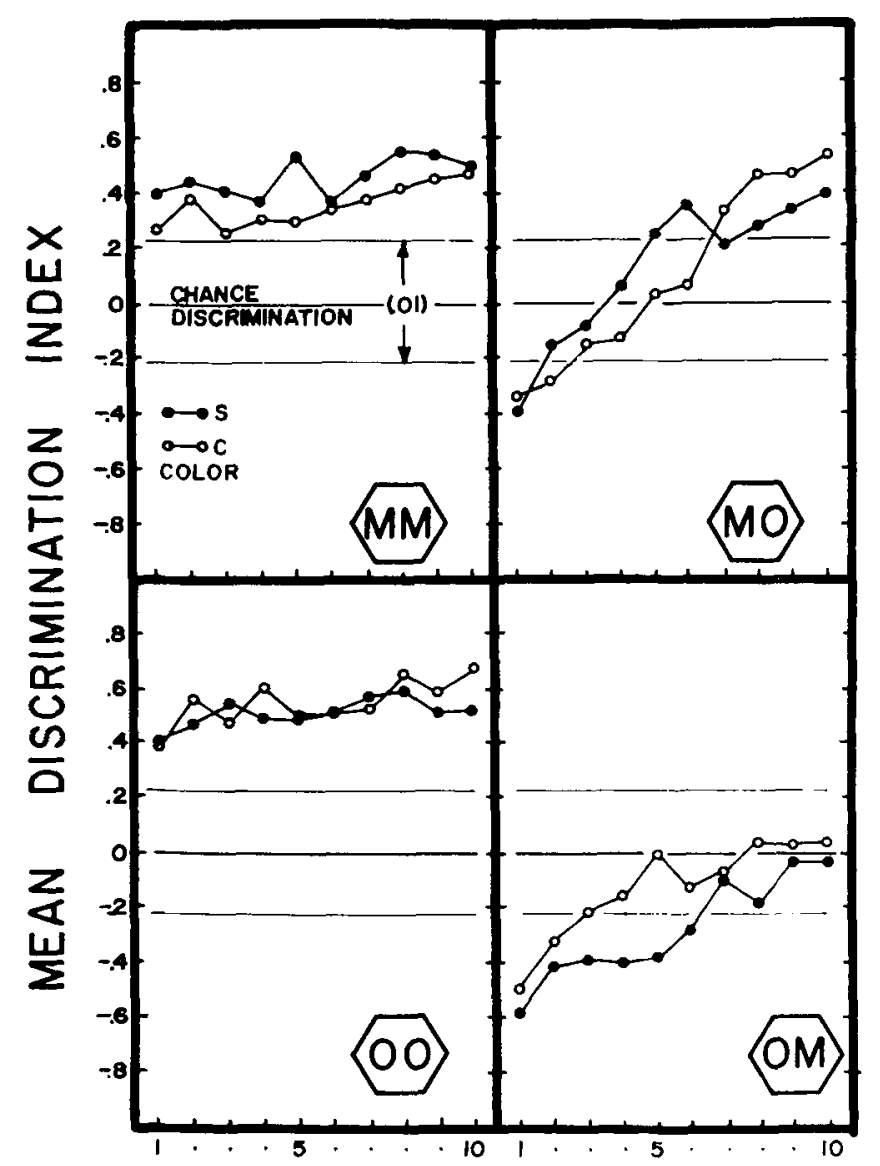

TRANSFER TRAINING in 3 DAY BLKS

Figure 4. The mean discrimination index (DI), in 3-day blocks, for all groups in transfer testing. Panels show groups with identical original-learning/transfer-testing criterion-contingency histories, but differing in having their signal colors the same (S) or changed (C) in transfer testing from those experienced in original learning. A .01 confidence interval from zero or no discrimination is incorporated into each panel.

significant main effects for blocks of training were observed, and all other significant effects were interactions. Following the initial analysis, appropriate partitions of the significant interactions were done to enable an interpretation of the data.

On the avoidance measure, the OOS and OOC groups did not differ or improve during transfer testing. Both the MMS and MMC groups significantly increased their avoidances with transfer testing $[F(9,216)=2.230$, $\mathrm{p}<.05]$ but did not differ. Similarly, the OMS and OMC groups did not differ, and both significantly increased their avoidances during transfer testing $[F(9,216)$ $=11.16, \mathrm{p}<.01]$. The only effect due to color change was found in the MOS and MOC groups comparison. Pair members did not differ, and both groups increased in avoidances during transfer testing $[\mathrm{F}(9,216)=30.71$, $\mathrm{p}<.01]$. However, an interaction between $\mathrm{S}$ vs. $\mathrm{C}$ color condition and days of transfer testing was also observed
$[F(9,216)=3.81, p<.011$. Comparisons between MOS and MOC groups for each 3-day block found the MOS group superior on the avoidance measure only on the fifth and sixth 3-day block $[\operatorname{ts}(240)=2.43$ and 2.15 , respectively, ps $<.05]$. No other effects or interactions were observed in the avoidance-data analysis.

A similar but not identical pattern of results was observed on the DI measure. Pairs of groups with similar criterion contingencies in original learning and transfer testing (MMS vs. MMC and OOS vs. OOC) did not differ, and did not improve in DI measure over transfer testing. An additional partition comparing the combined MM groups with the combined $O O$ groups also found no differences, which indicates equivalent matching and oddity conditional performance for these groups in transfer and is the only impression from the figures that is not confirmed by the statistical analysis. Pairs of groups with criterion contingencies shifted between original learning and transfer testing (MOS vs. MOC and OMS vs. OMC) showed significant DI improvement with transfer testing $[\mathrm{Fs}(9,216)=28.26$ and 10.82 , respectively, ps $<.01]$, but did not differ. In addition, a significant signal color (S vs. C) $\times$ days of transfer testing interaction for only the MOS vs. MOC comparison was observed $[F(9,216)$ $=2.85, \mathrm{p}<.01]$; however, an additional partition showed the MOS and MOC groups differing only on the sixth 3-day block $[\mathrm{t}(240)=2.47, \mathrm{p}<.05]$. A comparison of the combined MO groups against the combined OM groups found, as expected, a substantial difference due to the direction of shift, with the MO shift groups performing better on the DI measure than the OM shift groups $[F(1,24)=10.80, p<.01]$. Differences due to transfer testing $[F(9,216)=36.61, p<.01]$ and a shift direction $X$ days of transfer testing interaction were also observed $[F(9,216)=2.48, p<.01]$.

The TwR data were also analyzed. No differences or change over transfer testing was observed for the MMS ms. MMC or OOS vs. OOC pairs. However, for the pairs of groups with criterion contingencies shifted between original learning and transfer testing (MOS vs. MOC and OMS vs. OMC), significant increases with transfer testing over days $[\operatorname{Fs}(9,216)=9.22$ and 3.22 , respectively, ps $<.01]$ and signal color (S vs. C) $X$ days of transfer testing interactions $[\mathrm{Fs}(9,216)=5.18, \mathrm{p}<.01$, and $2.39, \mathrm{p}<.05$, respectively] were observed.

One additional analysis was completed. Carter and Eckerman (1975) criticized Zentall and Hogan's (1974) concept-learning conclusion with pigeons because the shifted and nonshifted birds did not differ on the 1st day of transfer testing. An additional 3-way [criterion in original learning ( $M$ vs. $O$ ), criterion in transfer testing ( $M$ vs. O), and signal-color conditions (S vs. C) for the lst day of transfer testing] ANOVA on the DI measure found only one very large effect, an interaction between original-learning and transfer-testing criterion-contingency conditions $[F(1,24)=82.207, p<.01]$. A partition of this interaction was accomplished by comparing pairs that had had similar criterion-contingency histories 
that differed only in transfer signal colors ( $\mathrm{S}$ vs. C). No differences between pairs with similar criterion-contingency histories were found [DI means for pairs: MMS (.31) vs. MMC (.23), OOS (.39) vs. OOC (.36), MOS $(-.47)$ vs. MOC $(-.48)$, OMS $(-.68)$ vs. OMC $(-.53)$; $\operatorname{ts}(24) ı=.48, .24, .04$, and .95 , respectively, all ps $>.05]$. Since like criterion group pairs did not differ, each pair was combined and tested against zero discrimination to determine if they differed from zero or chance discrimination on the first day of transfer testing. The combined $\mathrm{MM}$ and $\mathrm{OO}$ groups discriminated significantly above zero [mean DIs $=.27$ and .38 , respectively; $\operatorname{ts}(24)=3.41$ and 4.75 , respectively, $p<.01]$. The combined MO and OM groups, with their criterion contingencies shifted between original learning and transfer, showed significant negative transfer effects and dropped significantly below zero or chance discrimination levels [mean DIs $=-.47$ and -.60 , respectively; $\operatorname{ts}(24)=5.98$ and 7.61 , respectively, $p<.01]$. The identical result pattern was observed in an analysis of the first transfertest day's avoidance data, and is omitted for brevity. Additional analyses, on the number of shuttles per trial or the number of shuttles during the ITI, did not provide any useful information, either in original learning or transfer testing, and are omitted.

\section{GENERAL DISCUSSION}

The results of the present study are clear. Trained in a shuttlebox with the linear-presentation conditionaldiscrimination procedure, goldfish learn either a matching or oddity discrimination, learn it to avoid shock, and learn it at the conceptual level. The results meet all of the requirements for these conclusions. Performance in transfer is dependent on the relationship between the criterion contingencies in original learning and transfer testing and independent of the signal colors in transfer. When the criterion contingency is the same in original learning and transfer testing (MM, OO), transfer is positive. When there is a shift of criterion contingency between original learning and transfer testing (MO, OM), transfer is negative. Pairs of groups with the same criterion history (MM, OO, MO, or OM) perform comparably in transfer regardless of the fact that one member of each pair had the same (S) color signals it had experienced in original learning and the other had its signal colors completely changed (C) in transfer. Furthermore transfer effects, both positive and negative, occur on the 1 st day of transfer testing.

It is noteworthy that significant negative as well as positive transfer effects were observed. Both are required for the conceptual learning conclusion. Both represent a generalization of what was originally learned in response to the transfer-test situation. To grasp this, assume that the shift in criterion contingency between original learning and transfer testing is a reversal of reinforcement contingency. The effects of reversing shockpairing contingencies following acquisition of a conventional signal-specific discrimination are well known.
Significant negative transfer occurs. The effect of changing both signals following acquisition of a signal-specific discrimination is also known. Zero or chance discrimination occurs. Both these effects have been observed in goldfish (Zerbolio, 1981; Zerbolio \& Royalty, 1982). Changing the signals and reversing the shock pairing has no logical basis in the conventional discrimination procedure, because changing the signals creates an entirely new problem. With new signals, one signal-specific shockpairing arrangement is the logical equivalent of any other, and "reversal" no longer has any particular meaning. However, reversing (shifting) contingencies and changing signal colors does have meaning in the conditional discrimination design. When taught to conditionally discriminate, animals are expected to learn which relationships between signals (matching or oddity) are paired with shock rather than which specific signals are shock paired. The learned relationship between the members of one set of signals is expected to generalize to other signal sets. Thus, for groups with the same criterion relationships in original learning and transfer testing, when the transfer signals are the same as in original learning, positive transfer effects are expected. When the signals are changed in transfer testing, generalized positive transfer effects are expected. By the very same logic, when the criterion relationship is reversed in transfer testing (shifted), the negative-reversal transfer effect is expected. Thus, shifted groups with the same signals are expected to show the reversal negative transfer effect, and shifted groups with their signals changed as well are expected to show generalized negative transfer. This last effect is a generalization of the reversal phenomenon. The goldfish in the present study met all of these transfer and generalized-transfer expectations.

An important consideration for all of these transfer effects is the assumption that goldfish learn to discriminate between signals on the basis of color, and not on the basis of some other signal parameter. An alternative explanation might be to suggest that their discrimination was based on brightness differences. However, this seems unlikely. First, recall that, on the basis of measured luminances, the hurdle trial standard was always brighter than the response-selectable end-light sources (luminances ranges of 5 to $15 \mathrm{~mL}$ vs. 1 to $4 \mathrm{~mL}$, respectively). Second, signal sources were observed to vary as much between different sources of the same color as between sources of different color. Since goldfish were tested in transfer in a shuttlebox different from the one experienced in original learning, changing shuttleboxes would necessarily introduce random variability in the relative brightness of signal colors. Thus, one would expect substantially less consistency in transfer and generalization effects if goldfish had originally learned on the basis of brightness differences. The variability in brightness, both between sources of the same color and sources of different colors, appears to rule out any sort of brightness interpretation. If anything, the present results are remarkably consistent with a color-discrimina- 
tion analysis. However, not all of these results meet the predictions of this analysis. For instance, there is no reason to expect differences between oddity- and matching-criterion-trained animals. Yet, in original learning, it is clear that oddity-trained goldfish learn faster and reach higher conditional discrimination levels than do matching-trained goldfish. The same oddityover-matching superiority has been observed with other animals in positive-reinforcement conditional discrimination work (e.g., Zentall \& Hogan, 1974, 1978). Another unexpected finding is the asymmetry between the shifted MO and OM groups in learning the transfer-test task. Groups trained in transfer with the oddity procedure (MO) do better than those trained with the matching procedure (OM). It is clear that all four shifted groups suffer significant negative transfer due to the reversal of their criterion contingency in the initial phases of transfer testing. However, the MO-shifted groups learn the transfer task to levels above chance by the end of transfer testing, whereas the OM shifted groups do not. The OM groups reduce the negative-transfer impact of reversing their original-learning criterion and return to chance discrimination-level performances, but do not exceed chance during the 30 days of transfer testing. Note, however, that this asymmetry is consistent with the oddity-over-matching superiority observed in original learning. The last unexpected finding is the only statistically significant result involving signal-color change. Note that, between the sixth and seventh 3-day blocks, the relative positions of the same and changed MO groups reverse. This is obvious in the upper right panels of both Figures 3 and 4. The reasons for this color change $X$ days of transfer training interaction are not at all obvious and, at this time, can only be interpreted as a chance event.

In sum, then, it seems reasonable to conclude that goldfish learn a color-signal conditional discrimination at the conceptual level. By itself, the finding that conditional discrimination can be learned is not new, for several other laboratory animals have accomplished the task. What one tends to overlook is that goldfish learn the conditional discrimination to avoid shock. The differential reinforcers are shock and shock omission. As far as is known, only apes have been shown to be capable of mastering such a complex avoidance task (e.g., Rholes, Grunzke, \& Reynolds, 1963). Since goldfish learn conventional signal-specific discriminations to avoid shock (Zerbolio, 1981; Zerbolio \& Royalty, 1982; Zerbolio \& Wickstra, 1980), and as the present results show conditional discriminations as well, it seems that goldfish are the appropriate animal for use in the study of complex avoidance learning.

\section{REFERENCES}

Bowman, R., \& Sutherland, N. S. The discrimination of "W" and "V" shapes by goldfish. Quarterly Journal of Experimental Psychology, 1969, 21, 69-76.

Carter, D. E., \& Eckerman, D. A. Symbolic matching by pigeons: Rate of learning complex discrimination predicted from simple discriminations. Science, 1975, 187, 662-664.

Goldman, M., \& Shapiro, S. Matching-to-sample and oddityfrom-sample in goldfish. Journal of the Experimental Analysis of Behavior, 1979, 31, 259-266.

Horner, J. L., Longo, N., \& Bitterman, M. E. A shuttlebox for fish and a control circuit of general applicability. American Journal of Psychology, 1961, 74, 114-120.

Mackintosh, N. J. The psychology of animal learning. London: Academic Press, 1974.

Rholes, F. H., Grunzke, M. E., \& ReYnolds, H. H. Chimpanzee performance during the ballistic and orbital project Mercury flights. Journal of Comparative and Physiological Psychology, 1963, 56, 2-10.

Wickstra, L. L. Matching-to-sample and oddity-to-sample discrimination acquisition in the goldfish based upon avoidance contingencies. Unpublished doctoral dissertation, University of Missouri-St. Louis, 1980.

Zentall, T. R., \& Hogan, D. E. Abstract concept learning in the pigeon. Journal of Experimental Psychology, 1974, 102, 393-398.

Zentall, T. R., \& Hogan, E. Same/different concept learning in the pigeon: The effect of negative instance and prior adaptation to transfer stimuli. Journal of the Experimental Analysis of Behavior, 1978, 30, 177-186.

ZERBolio, D. J., JR. Discrimination avoidance learning and reversal by goldfish in a shuttlebox using a linear presentation procedure. Animal Learning \& Behavior, 1981, 9, 346-356.

Zerbolio, D. J., \& Royalty, J. L. Acquisition and extinction of differential responses to signals paired with shock or shock omission in goldfish: Evidence for truly discriminated avoidance learning. Animal Learning \& Behavior, 1982, 10, 377-389.

Zerbolio, D. J., JR., \& Wickstra, L. L. Instrumentally based conditioned avoidance response acquisition in goldfish in a simultaneous presentation task. Bulletin of the Psychonomic Society, 1979, 13, 311-313.

Zerbolio, D. J., JR., \& Wickstra, L. L. Instrumental avoidance acquisition by goldfish in a $Y$-maze using explicit and response-contingent cues. Animal Learning \& Behavior, 1980, 8, 304-310.

(Manuscript received October 12, 1982; revision accepted for publication March 20, 1983.) 